\title{
Caracterización de materiales a través de medidas de microscopía electrónica de barrido (SEM)
}

\author{
Josué Clavijo* \\ Universidad de América. Bogotá - Colombia
}

FeCha DE ENTREGA: 2 DE OCTUBRe DE 2012

FECHA DE APROBACIÓN: 7 DE MARZO DE 2013

\begin{abstract}
Resumen La microscopía de barrido electrónico (SEM) es una técnica de obtención de imágenes ampliamente utilizada en investigación de materiales debido a su alta resolución y capacidades de analizar características morfológicas, estructurales y químicas de las muestras bajo estudio. En este documento revisamos los fundamentos tanto conceptuales como de funcionamiento y utilidad de las imágenes que resultan de una medida de $S E M$, relacionando todo con el material más usado en fotocatálisis heterogénea, el dióxido de titanio $\left(\mathrm{TiO}_{2}\right)$.
\end{abstract}

\begin{abstract}
S E M$ is an image generation technique widely used on materials researchdue its high-resolution capabilities and proven aptitude to unveil morphological, structural and even chemical features of a given sample. This paper brings out a concise review of the fundamentals, instrumental details and usefulness of the resulting SEM images. All of the concluding facts were thus related with the most important photocatalyst: $\mathrm{TiO}_{2}$.

Palabras Clave: SEM, microscopía de barrido electrónico, fotocatálisis, $\mathrm{TiO}_{2}$.
\end{abstract}

Keywords: SEM, photocatalysis, image analysis, $\mathrm{TiO}_{2}$.

* Profesor investigador de la Universidad de América. Grupo de investigación: Grupo de Materiales Fotoactivos. josue.clavijo@gmail.com 


\section{Introducción}

La microscopía electrónica de barrido $S E M$ (de sus iniciales en el idioma inglés Scanning Electron Microscopy) es una técnica de análisis topográfico, estructural y composicional, la cual se ha usado extensamente en el estudio de tópicos tales como semiconductores [1]-8], nanopartículas [9, modificación de productos comerciales [10], catálisis hetereogénea [11]-[13], dispositivos de juntura p-n [14], películas delgadas [15], física de nanocomposiciones [16], fotoelectroquímica [17], dadas sus posibilidades de resolución, magnificación y versatilidad de variantes que permite que sea aplicada a diversidad de muestras, ambientes y necesidades experimentales (procesos térmicos, corrosivos, etc.).

En términos generales, un equipo de $S E M$ es capaz de tomar una "imagen" de la muestra aunque realmente lo que se detecta es la respuesta del material al impacto de un haz de electrones (electrones llamados primarios), en vez de registrar fotones propiamente dichos (como en la microscopía óptica). Al ser impactada por el haz de electrones de alta energía, la muestra produce una serie de señales que son registradas en los diferentes detectores del equipo, en donde cada una de dichas señales ofrece independientemente información acerca de la topografía, composición y hasta conductividad eléctrica de la muestra. Por supuesto, para adquirir la "imagen" se hace mover el haz en el plano $x y$ (con sistemas ópticos, antes de impactar la muestra) de forma que recorra el área deseada o planificada. La resolución que puede alcanzarse; es decir, la capacidad de distinguir en la imagen entre dos puntos diferentes pero próximos entre sí, en la superficie del material, puede ser tan alta como $2 \mathrm{~nm}[18$.

En términos de lo que se puede estudiar con $S E M$, se puede afirmar que los resultados de una análisis por $S E M$ se resumen en información topográfica, morfológica, de composición química, de cristalografía (estructura y orientación de grano) y de dinámica de reacciones (experimentos in situ).

\section{SEM: Instrumental y teoría}

\subsection{Instrumental: ¿Cómo funciona un SEM?}

La idea básica de funcionamiento de un equipo de $S E M$ es la siguiente: se genera un haz de electrones de alta energía (o electrones incidentes $E I$ ) y se usa un sistema de lentes para focalizar el haz y hacerlo incidir sobre una muestra, la cual generará, a su vez, electrones (llamados secundarios y retrodispersados con siglas $S E$ y $B S E$, respectivamente) como respuesta al impacto electrónico, y dichos electrones que salen de la muestra son detectados mediante dispositivos EverhartThornley y SED (Solid State Detector) que registran cada uno la cantidad de electrones detectados y lo convierten en una señal digital que se interpreta como intensidad de color, para construir una "imagen" aunque no se usen fotones. Si, además de lo anterior, se tiene todo el equipo trabajando en una atmósfera interna prácticamente inexistente (alto vacío), y se reemplaza el detector de los SE convencional (Everhart-Thornley) por uno que genera un campo eléctrico para llevar los SE hacia el cuerpo del detector, entonces se puede hacer ESEM 
(environmental SEM) o SEM ambiental, el cual es una técnica muy usada en la actualidad para poder tomar imágenes de muestras "difíciles" de tratar: a) muestras aislantes, b) sensibles al vacío o radiación, c) muestras en estado líquido, además de dar la posibilidad de estudiar procesos in situ tales como corrosión, estrés mecánico o hidratación/deshidratación. La Figura 1 presenta un esquema del equipo, y la Figura 2 ejemplifica cómo se genera la "imagen" (en adelante, se usará el término imagen sin comillas, dando por explicado que aunque no proviene de una señal de intensidad o flujo de fotones, el resultado final es una interpretación en escala de colores del flujo de electrones $S E$ o $B S E$ detectados).

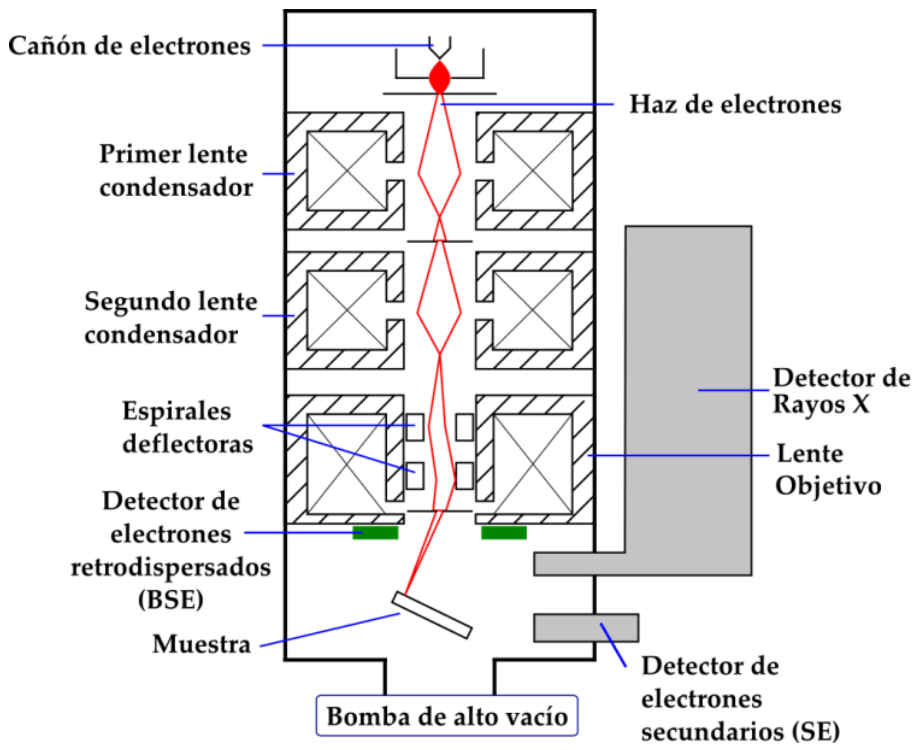

Figura 1. Esquema de un equipo de $S E M$.

Los dispositivos para generar el haz de electrones son generalmente de dos tipos: Emisores termoiónicos o los cañones de emisión de campo (FEG, Field Emission Gun) [19. Los termoiónicos generan electrones de alta velocidad (y, por ende, alta energía) cuando se calienta un filamento metálico (generalmente tungsteno) o de un compuesto como $\mathrm{LaB}_{6}$ si el sistema está en alto vacío. Un tipo más reciente de emisores de electrones los $F E G$, funcionan cuando una punta cristalina de tungsteno recubierta de óxido de Zirconio se somete a un gran campo eléctrico (del orden de los $\mathrm{kV} / \mathrm{cm}$. Todos ellos requieren un ambiente de alto vacío (del orden de $10^{-6}$ torr) para minimizar la corrosión del emisor, que está a alta temperatura, ya que puede reaccionar con las moléculas del aire. 


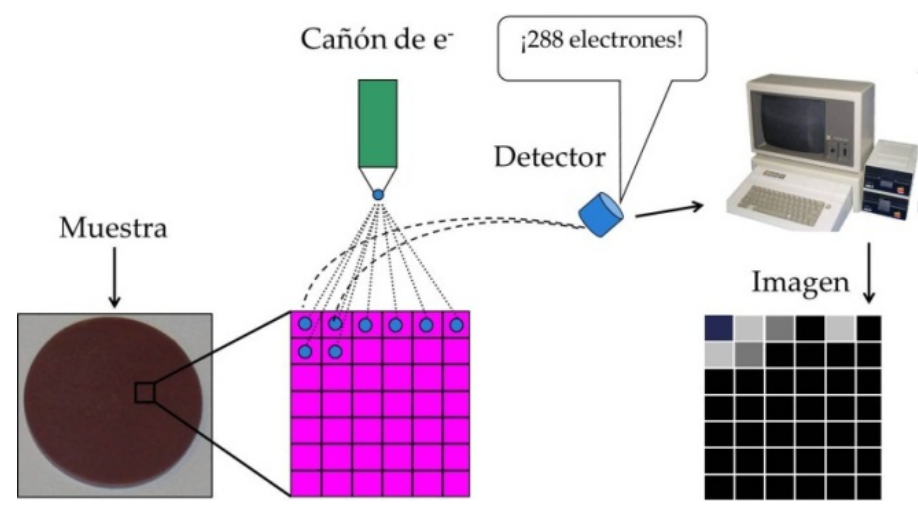

Figura 2. Secuencia del proceso de formación de la imagen. () y ${ }^{\circledR}$ Harald Fjeld, University of Oslo (Noruega).

\subsection{Teoría: Interacción electrones-materia}

Cuando el haz de electrones impacta la muestra, se generan dos tipos de señales: los electrones Secundarios $(S E)$ y los electrones retrodispersados $(B S E)$. Cada una de esas señales brinda información diferente acerca de la muestra, dado que se generan a partir de procesos diferentes. Como se ilustra en la figura 3 , los electrones $S E$ se generan por la colisión de los $E I$ con los átomos superficiales de la muestra (los primeros cristales); son electrones de baja energía ( 10-50 eV), y como provienen de la superficie pueden escapar con información de la morfología de la muestra (información topográfica). Los $S E$ son mayores en número que los $E I$, por lo cual se puede saber que no son simplemente electrones $E I$ reflejados que no interactúan con la muestra. Ahora bien, puede haber electrones $S E 1$ y $S E 2$ ya que algunos electrones $E I$ pueden viajar al interior de la muestra y colisionar con átomos que no necesariamente estén en la superficie sino unas capas atómicas debajo, probablemente (electrones SE2).

Por su lado, los electrones BSE son electrones que alcanzan a viajar mucho más al interior de la muestra y, aunque no choquen con ningún átomo, traen información acerca de la muestra debido a que son perturbados en su viaje por los campos magnéticos de los núcleos atómicos de la muestra y pueden ser desviados con ángulos mayores de $180^{\circ}$, de forma que puedan escapar de la superficie (Figura 3).

\subsection{Acerca de la formación de la imagen: Resolución y magnificación}

Como se afirmó anteriormente, la imagen se forma de manera digital. Un PC con el software adecuado interpreta la cantidad de electrones detectados (ya sean $S E$ o $B S E)$ y a cada zona le asigna una intensidad de color en la escala de grises de acuerdo a una convención general: Las zonas de donde se detecten mayor 


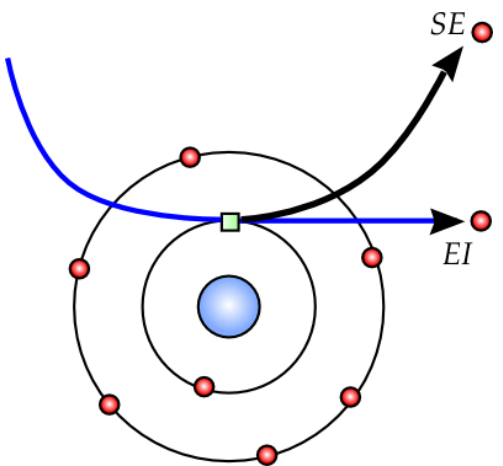

(a)

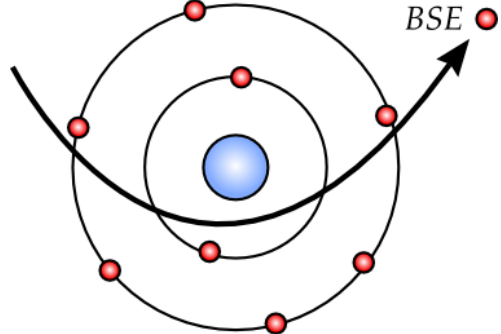

(b)

Figura 3. Generación de electrones $S E$ (izquierda) y $B S E$. Tomado bajo licencia CC-BY de wikimedia commons.

número de electrones se representarán con un color más blanco. Por ello, las imágenes construidas tienen la apariencia de una fotografía en escala de grises, aunque algunas veces se añade un color en escala de saturación creciente para resaltar ciertos aspectos morfológicos o para resaltar cambios en la superficie de la muestra.

Resolución y magnificación. Los términos conocidos como resolución y magnificación suelen tomarse como sinónimos aunque presentan diferencias. Al hablar de la resolución de una imagen de $S E M$ se tiene en cuenta la capacidad conjunta del sistema haz de electrones - sistema óptico - detectores - software de adquisición e interpretación para brindar una distinción clara entre dos puntos cercanos dentro del área de muestra en la cual se está tomando el barrido. Generalmente, a menor diámetro del haz de electrones incidentes EI, mayor será la resolución; ya que se barrerá un área muy pequeña $\left(\sim \mathrm{nm}^{2}\right)$ con respecto a toda el área a la cual se le hace incidir el haz.

La magnificación, por su parte, está relacionada al tiempo con el área de muestra que se barre con el haz de electrones $E I$ y con el área de visualización que se construya en el software de interpretación. Principalmente se puede variar la magnificación desde 10 y hasta 500.000 veces el tamaño real de un área de muestra, dependiendo de los dos parámetros antes mencionados. Así, la mayor magnificación se logrará barriendo un área muy pequeña e interpretando la señal para construir una imagen de grandes dimensiones (pantalla o visualización grande, de $1920 \times 1080$ pixel, por ejemplo.)

\section{SEM: Caracterización de materiales}

Dada la alta resolución que se puede lograr en $S E M$, es posible conocer las características más detalladas de un material: su estructura cristalina, porosidad, 
morfología superficial y hasta su composición química. Es de aclarar que las imágenes de electrones $S E$ tienen mucha mejor resolución que las generadas con electrones $B S E$, debido a el número de electrones detectados, que es mucho mayor en los $S E$, y a efectos de superficie y penetración de los electrones incidentes o EI. Algunos ejemplos de imágenes de $S E M$ se presentan en la Figuras 4 a 8 . Se puede apreciar en las distintas figuras que la morfología y la estructura cristalina de cada muestra analizada es particularmente distinguible, siendo estructuras muy variadas en formas y disposiciones; la principal característica morfológica de todas las muestras (todas ellas de materiales semiconductores) es su altísima área superficial, la cual es un parámetro muy importante; ya que la mayoría de procesos de transporte tanto eléctrico como de catálisis asistida por luz están muy fuertemente influenciados por la superficie de material.

Nótese cómo, especialmente en el caso del $\mathrm{TiO}_{2}$, los semiconductores presentan formas muy repetitivas y armónicas, algunas de ellas hasta espectaculares, lo cual es una evidencia directa del carácter cristalino de su crecimiento (es decir, que crecen formando millones de cristales con la misma estructura y morfología). Cuando no se obtiene mediante un proceso de síntesis de materiales la forma casi esférica que presenta el $\mathrm{TiO}_{2}$ de la Figuras 4 a 8 se puede perder área superficial ya que la forma esférica es la que mejor relación área/volumen presenta.

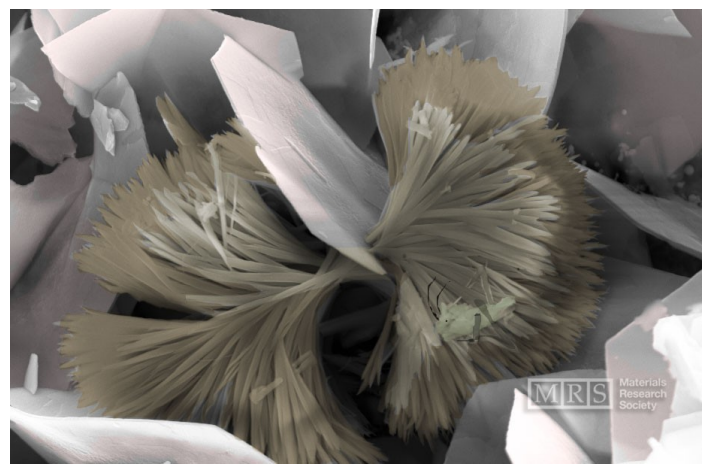

Figura 4. Imágenes SEM: Nanocristales de $\mathrm{CuInS}_{2}$. (ㅇ y ${ }^{\circledR}$ Materials Research Society.

Por otro lado, y como se ilustra también en la figura 9 la composición química se puede diferenciar según los diferentes patrones de contraste que cada zona, según los átomos que la conforman, origina. Frecuentemente, las imágenes obtenidas se relacionan con los resultados de otras técnicas y se contrastan contra modelos estructurales [20]-35] para estudiar a una mayor profundidad un material; con ello el $S E M$ ha logrado incursionar en campos de la ciencia tan amplios como los estudios de topografía [36], recubrimientos microestructurados [37], paleobotánica [38, reconstrucción de imágenes en 3D [39], nanoelectricidad [40], fotoluminiscencia y emisión de campo [41, síntesis solvotérmica [42, sensores de gases 


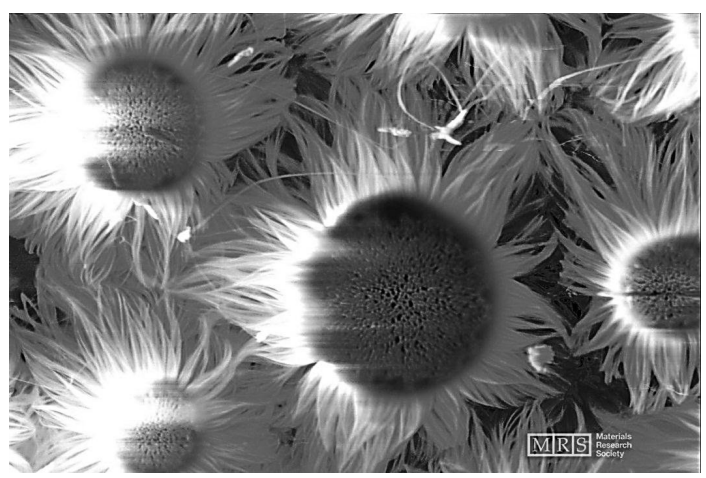

Figura 5. Imágenes SEM: Nanoestructuras de $\mathrm{SiO}_{\mathrm{x}}$. (이 y ${ }^{\circledR}$ Materials Research Society.

43, nanobarras (nanorods) 44, nanoalambres (nanowires) 45, fotocatálisis [46]- 48, semiconductores compuestos [49], computación por software de imagen [50], geomateriales [51], nanoesferas (nanospheres) [52], nanoestructuras [53]- 55], sensores de glucosa [56], compuestos antibacterianos [57], electroquímica de la corrosión [58], síntesis química por Spray Pyrolysis y por evaporación térmica [5960; películas delgadas dopadas 61].

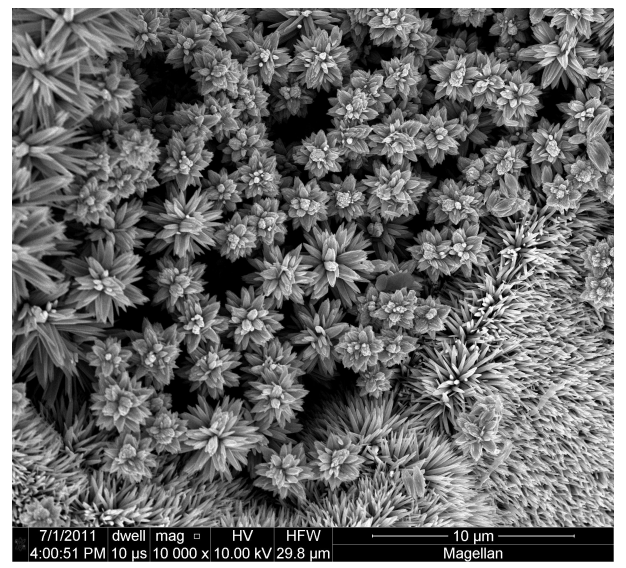

Figura 6. Imágenes SEM: Nanocristales de ZnO. 이 y ${ }^{\circledR}$ Magellan Institute. 


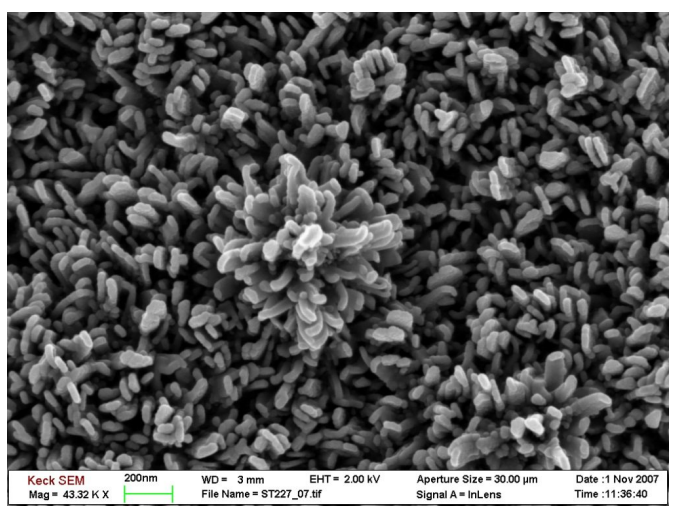

Figura 7. Imágenes SEM: Nanoestructuras de $\mathrm{TiO}_{2}$. ㅇ y ${ }^{\circledR}$ Keck Institute.

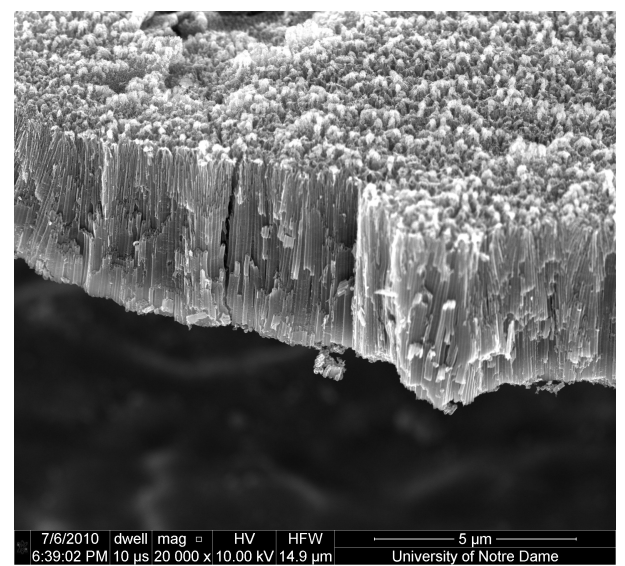

Figura 8. Imágenes SEM: Nanotubos de $\mathrm{TiO}_{2}-\mathrm{SrTiO}_{3}$. 이 y ${ }^{\circledR}$ University of Notre Dame.

\section{Conclusiones}

En este documento se presentaron, de manera básica, los fundamentos de la técnica de análisis morfológico, estructural y química llamada Microscopía de barrido electrónico o SEM. Se explicó el funcionamiento y las diferentes señales que una muestra origina, así como la información que cada una de ellas conlleva. Se aclaran aspectos experimentales como la resolución y la magnificación y, finalmente, se presentan ejemplos claros de los resultados de la técnica en términos de morfología, estudios de nanoestructuras y análisis químico elemental. 


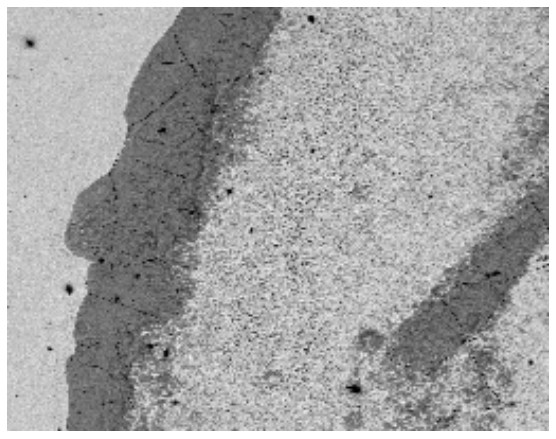

(a) Aleación

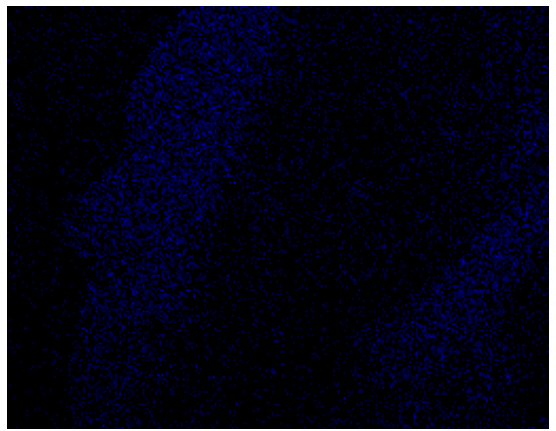

(c) $\mathrm{Fr}$

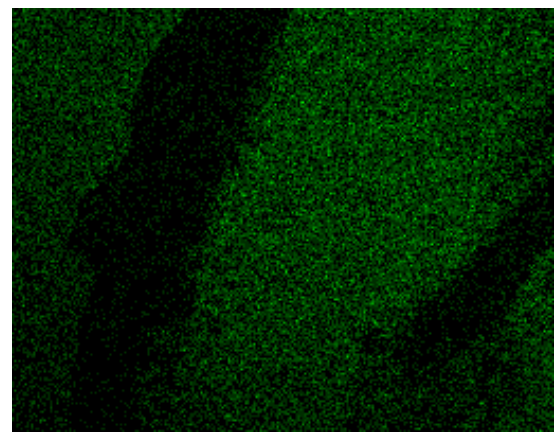

(b) $\mathrm{Ce}$

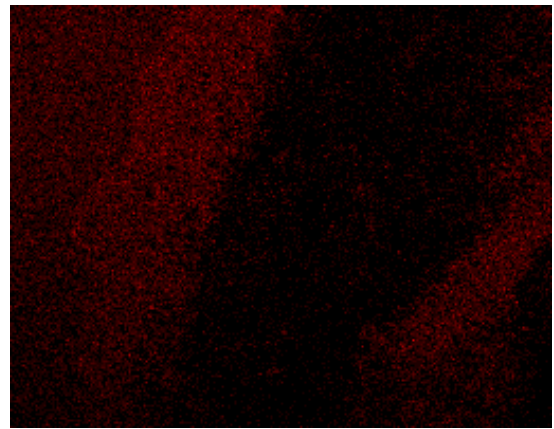

(d) $\mathrm{Sr}$

Figura 9. Análisis de composición química en una aleación de Fe-Ce-Sr. @ y ${ }^{\circledR}$ Harald Fjeld, University of Oslo (Noruega).

Agradecimientos: El autor desea agradecer a Dios, en primera medida, por su amor, apoyo y presencia. A la Fundación Universidad de América por facilitar el escenario propicio para la realización del primer Seminario de Fotocatálisis. Al director del grupo de Fotocatálisis de la Universidad de América, Dr. Sc. César Quiñones, por su apoyo y sus valiosas sugerencias para la realización de este documento.

\section{Referencias}

1. Mahajan L.H., Mhaske S.T.: Composite microspheres of poly(o-anisidine)/ $/ \mathrm{TiO}_{2}$, Materials Letters 68 183-186. (2012)

2. Murcia-López S. et al.: Photocatalytic activity of single and mixed nanosheetlike Bi2WO6 and $\mathrm{TiO}_{2}$ for Rhodamine $\mathrm{B}$ degradation under sunlike and visible illumination, Applied Catalysis A: General 423-424 34-41 (2012)

3. Xiuwen Cheng et al.: Enhanced Photocatalytic Activity of Nitrogen Doped $\mathrm{TiO}_{2}$ Anatase Nano-Particle under Simulated Sunlight Irradiation, Energy Procedia 16 $598-605(2012)$ 
4. Sangchay W. et al.: Comparison of photocatalytic reaction of commercial P25 and synthetic $\mathrm{TiO}_{2}-\mathrm{AgCl}$ nanoparticles, Procedia Engineering 32590 - 596 (2012)

5. Henderson M.A.: A surface science perspective on $\mathrm{TiO}_{2}$ photocatalysis, Surface Science Reports 66 185-297 (2011)

6. Penghua Wang et al.: Carbon-sensitized and nitrogen-doped $\mathrm{TiO}_{2}$ for photocatalytic degradation of sulfanilamide under visible-light irradiation, Water Research 455015 -5026 (2011)

7. Wu F. et al.: Membrane vis-LED photoreactor for simultaneous penicillin G degradation and $\mathrm{TiO}_{2}$ separation, Powder Technology 213 192-198 (2011)

8. Faure M. et al.: Study of photocatalytic damages induced on E. coli by different photocatalytic supports (various types and $\mathrm{TiO}_{2}$ configurations), Journal of Photochemistry and Photobiology A: Chemistry 222 323-329 (2011)

9. Ubonchonlakate K., Sikong L., Saito F.: Photocatalytic disinfection of P.aeruginosa bacterial Ag-doped $\mathrm{TiO}_{2}$ film, Procedia Engineering, Volumen 32, Páginas 656-662 (2012)

10. Chengbao Liu, Zhigang Chen, Zhi Miao, Feng Chen, Cheng Gu, Minqi Huang, Xiaobing Zhao,: Properties and preparation of porous carbon material supported with modified $\mathrm{TiO}_{2}$, Procedia Engineering, Volumen 27, Páginas 557-563 (2012)

11. Magdalena Palacio, Paula I. Villabrille, Gustavo P. Romanelli, Patricia G. Vázquez, Carmen V. Cáceres, Preparation, characterization and use of $\mathrm{V}_{2} \mathrm{O}_{5}-\mathrm{TiO}_{2}$ mixed xerogels as catalysts for sustainable oxidation with hydrogen peroxide of 2,3,6trimethylphenol, Applied Catalysis A: General, Volúmenes 417-418, 29 de Febrero de 2012, Páginas 273-280 (2012)

12. K.V. Narayana, A. Venugopal, K.S. Rama Rao, S. Khaja Masthan, V. Venkat Rao,

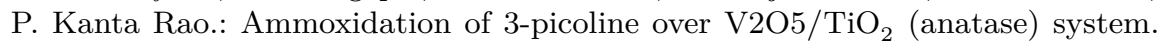
II. Characterisation of the catalysts by DTA, SEM, FTIR, ESR and oxygen and ammonia chemisorption, Applied Catalysis A: General, Volumen 167, Número 1, 5 de Febrero de 1998, Páginas 11-22 (1998)

13. Won-Chun Oh, Feng-Jun Zhang, Ming-Liang Chen, Synthesis and characterization of $\mathrm{V}-\mathrm{C} 60 / \mathrm{TiO}_{2}$ photocatalysts designed for degradation of methylene blue, Journal of Industrial and Engineering Chemistry, Volumen 16, Número 2, 25 de Marzo de 2010, Páginas 299-304 (2010)

14. Shifu Chen, Wei Zhao, Wei Liu, Sujuan Zhang.: Preparation, characterization and activity evaluation of p-n junction photocatalyst p- $\mathrm{ZnO} / \mathrm{n}-\mathrm{TiO}_{2}$, Applied Surface Science, Volumen 255, Número 5, Parte 1, 30 de Diciembre de 2008, Páginas 2478-2484 (2008)

15. S.C. Lee, Huogen Yu, Jiaguo Yu, C.H. Ao.: Fabrication, characterization and photocatalytic activity of preferentially oriented $\mathrm{TiO}_{2}$ films, Journal of Crystal Growth, Volumen 295, Número 1, 15 de Septiembre de 2006, Páginas 60-68 (2006)

16. Jiabin Zhou, Ya Cheng, Jiaguo Yu.: Preparation and characterization of visiblelight-driven plasmonic photocatalyst $\mathrm{Ag} / \mathrm{AgCl} / \mathrm{TiO}_{2}$ nanocomposite thin films, Journal of Photochemistry and Photobiology A: Chemistry, Volumen 223, Números 2-3, 25 de Septiembre de 2011, Páginas 82-87. (2011)

17. Remigijus Juäkänas, Darius Aviänis, Putinas Kalinauskas, Algirdas Selskis, Raimondas Giraitis, Vidas Pakätas, Violeta Karpaviàienà, Stasà Kanapeckaità, Zenius Mockus, Rokas Kondrotas.: XRD, SEM and photoelectrochemical characterization of ZnSe electrodeposited on $\mathrm{Cu}$ and $\mathrm{Cu}$-Sn substrates, Electrochimica Acta, Volumen 70, 30 de Mayo de 2012, Páginas 118-123 (2012)

18. Vilá A., Comerma A., Trenado J., Arbat A., Gascon D., Garrido L., Diáguez A.: SEM/FIB for characterization of nanosized imagers, Procedia Engineering, Volumen 5, Páginas 697-700. (2010) 
19. Perovic D.D., Castell M.R., Howie A., Lavoie C., Tiedje T., Cole J.S.W.: Fieldemission SEM imaging of compositional and doping layer semiconductor superlattices, Ultramicroscopy, Volumen 58, Número 1, Páginas 104-113 (1995)

20. Edward T. Yu.: Nanoscale characterization of semiconductor materials and devices using scanning probe techniques, Materials Science and Engineering: R: Reports, Volumen 17, Números 4-5, 15 de Noviembre de 1996, Páginas 147-206 (1996)

21. Edwards N.V., Bremser M.D., Batchelor A.D., Buyanova I.A., Madsen L.D., Yoo S.D., Wethkamp T., Wilmers K., Cobet C., Esser N., Davis R.F., Aspnes D.E., Monemar B.: Optical characterization of wide bandgap semiconductors, Thin Solid Films, Volumen 364, Números 1-2, 27 de Marzo de 2000, Páginas 98-106 (2000)

22. Mane R.S, Lokhande C.D.: HRTEM, SEM and XRD characterization of nanocrystalline $\mathrm{Sb}_{2} \mathrm{~S}_{3}$ thin films deposited by chemical bath route, Surface and Coatings Technology, Volumen 172, Número 1, 15 de Julio de 2003, Páginas 51-56 (2003)

23. Analá L. Soldati, Laura Baquio, Horacio Troiani, Carlos Cotaro, Anja Schreiber, Alberto Caneiro, Adriana Serquis.: High resolution FIB-TEM and FIB-SEM characterization of electrode/electrolyte interfaces in solid oxide fuel cells materials, International Journal of Hydrogen Energy, Volumen 36, Número 15, Julio de 2011, Páginas 9180-9188 (2011)

24. Zahava Barkay, Ilia Rivkin, Rimona Margalit.: Three-dimensional characterization of drug-encapsulating particles using STEM detector in FEG-SEM, Micron, Volumen 40, Número 4, Junio de 2009, Páginas 480-485 (2009)

25. Nakabayashi D., Silva P.C., Ugarte D.: Inexpensive two-tip nanomanipulator for a SEM, Applied Surface Science, Volumen 254, Número 1, 31 de Octubre 2007, Páginas 405-411 (2007)

26. Robert Krueger.: Dual-column (FIB-SEM) wafer applications, Micron, Volumen 30, Número 3, Junio de 1999, Páginas 221-226 (1999)

27. Mätois J.J., Le Lay G.: Complementary data obtained on the metal-semiconductor interface by LEED, AES and SEM: Pb/Ge(111), Surface Science, Volumen 133, Números 2-3, 2 de Octubre de 1983, Páginas 422-442 (1983)

28. Baykul M.C, Balcioglu A.: AFM and SEM studies of CdS thin films produced by an ultrasonic spray pyrolysis method, Microelectronic Engineering, Volúmenes 51-52, Mayo de 2000, Páginas 703-713 (2000)

29. Hirata T., Akane T., Jinno S., Kuno T., Yang Y., Fujiwara Y., Nakamura A., Takeda Y.: SEM observation of InP/ErP/InP double heterostructures grown on $\operatorname{InP}(001), \operatorname{InP}(111) A$, and $\operatorname{InP}(111) B$, Materials Science in Semiconductor Processing, Volumen 6, Números 5-6, Octubre-Diciembre de 2003, Páginas 473-476 (2000)

30. Petersen C., Lasagni A., Holzapfel C., Daniel C., Mäcklich F., Veith M.: SEM/TEM characterization of periodical novel amorphous/nano-crystalline micro-composites obtained by laser interference structuring: The system $\mathrm{HAlO}-\mathrm{Al} \cdot \mathrm{Al}_{2} \mathrm{O}_{3}$, Applied Surface Science, Volumen 253, Número 19, 31 de Julio de 2007, Páginas 8022-8027 (2007)

31. David A. Stanley, Hossein Alizadeh, Amr Helmy, Nazir P. Kherani, Li Qian, Stefan Zukotynski.: SEM-mapped micro-photoluminescence studies of highly luminescent micro-clusters in erbium-doped silicon-rich silicon oxide, Journal of Luminescence, Volumen 131, Número 1, Enero de 2011, Páginas 72-77 (2011)

32. Iacob E., Bersani M., Lui A., Giubertoni D., Barozzi M., Anderle M.: Topography induced by sputtering in a magnetic sector instrument: an AFM and SEM study, Applied Surface Science, Volumen 238, Números 1-4, 15 de Noviembre de 2004, Páginas 24-28 (2011) 
33. Robert-Goumet C., Monier G., Zefack B., Chelda S., Bideux L., Gruzza B., Awitor O.K.: SEM and XPS studies of nanohole arrays on $\operatorname{InP}\left(\begin{array}{lll}1 & 0 & 0\end{array}\right)$ surfaces created by coupling AAO templates and low energy $\mathrm{Ar}^{+}$ion sputtering, Surface Science, Volumen 603, Número 19, 1 de Octubre de 2009, Páginas 2923-2927 (2009)

34. Hui Luo, L.E. Scriven, Lorraine F. Francis.: Cryo-SEM studies of latex/ceramic nanoparticle coating microstructure development, Journal of Colloid and Interface Science, Volumen 316, Número 2, 15 de Diciembre de 2007, Páginas 500-509 (2007)

35. Olivier Guise, Carl Strom, Nisha Preschilla.: STEM-in-SEM method for morphology analysis of polymer systems, Polymer, Volumen 52, Número 5, 1 de Marzo de 2011, Páginas 1278-1285 (2011)

36. Iacob E., Bersani M., Lui A., Giubertoni D., Barozzi M., Anderle M.: Topography induced by sputtering in a magnetic sector instrument: an AFM and SEM study, Applied Surface Science, Volumen 238, Números 1-4, 15 de Noviembre de 2004, Páginas 24-28, ISSN 0169-4332 (2004)

37. Hui Luo, L.E. Scriven, Lorraine F. Francis.: Cryo-SEM studies of latex/ceramic nanoparticle coating microstructure development, Journal of Colloid and Interface Science, Volumen 316, Número 2, 15 De Diciembre de 2007, Páginas 500-509 (2007)

38. Imogen Poole, Geoffrey E. Lloyd.: Alternative SEM techniques for observing pyritised fossil material, Review of Palaeobotany and Palynology, Volumen 112, Número 4, De Noviembre de 2000, Páginas 287-295 (2000)

39. Franziska Schmidt, Markus KÃijhbacher, Ulrich Gross, Antonius Kyriakopoulos, Helmut Schubert, Rolf Zehbe.: From 2D slices to 3D Volumens: Image based reconstruction and morphological characterization of hippocampal cells on charged and uncharged surfaces using FIB/SEM serial sectioning, Ultramicroscopy, Volumen 111, Número 4, De Marzo de 2011, Páginas 259-266 (2011)

40. Muhamamd Naeem Ashiq, Muhammad Fahad Ehsan, Muhammad Javed Iqbal, Iftikhar Hussain Gul.: Synthesis, structural and electrical characterization of $\mathrm{Sb}_{3}^{+}$ substituted spinel nickel ferrite ( $\mathrm{NiSbxFe} 2-\mathrm{xO} 4)$ nanoparticles by reverse micelle technique, Journal of Alloys and Compounds, Volumen 509, Número 16, 21 De Abril de 2011, Páginas 5119-5126 (2011)

41. Ghulam Nabi, Chuanbao Cao, Waheed S. Khan, Sajad Hussain, Zahid Usman, Tariq Mahmood, Noor Abass Din Khattak, Suling Zhao, Xu Xin, Dapeng Yu, Xuewen Fu.: Synthesis, characterization, photoluminescence and field emission properties of novel durian-like gallium nitride microstructures, Materials Chemistry and Physics, Volumen 133, Números 2-3, 16 De Abril de 2012, Páginas 793-798 (2012)

42. Juan Yang, Guoxiu Wang, Hao Liu, Jinsoo Park, Xiaonong Cheng.: Controlled synthesis and characterization of ZnSe nanostructures via a solvothermal approach in a mixed solution, Materials Chemistry and Physics, Volumen 115, Número 1, 15 May 2009, Páginas 204-208 (2009)

43. Balamurugan C., Vijayakumar E., Subramania A.: Synthesis and characterization of $\mathrm{InNbO}_{4}$ nanopowder for gas sensors, Talanta, Volumen 88, 15 De Enero de 2012, Páginas 115-120 (2012)

44. Maleki M., Mirdamadi Sh., Ghasemzadeh R., Sasani Ghamsari M.: Preparation and characterization of cadmium sulfide nanorods by novel solvothermal method, Materials Letters, Volumen 62, Números 12-13, 30 De Abril de 2008, Páginas 1993-1995 (2008)

45. Emanuela Filippo, Gioacchino Micocci, Antonio Tepore, Tiziana Siciliano.: Fabrication of $\alpha-\mathrm{TeO}_{2}$ smooth and beaded microwires by thermal evaporation method, Journal of Crystal Growth, Volumen 336, Número 1, 1 De Diciembre de 2011, Páginas 101-105 (2011) 
46. Won-Chun Oh, Feng-Jun Zhang, Ming-Liang Chen.: Synthesis and characterization of $\mathrm{V}-\mathrm{C}_{60} / \mathrm{TiO}_{2}$ photocatalysts designed for degradation of methylene blue, Journal of Industrial and Engineering Chemistry, Volumen 16, Número 2, 25 De Marzo de 2010, Páginas 299-304 (2010)

47. Chen Shifu, Yang Yunguang, Liu Wei.: Preparation, characterization and activity evaluation of $\mathrm{TiN} / \mathrm{F}-\mathrm{TiO}_{2}$ photocatalyst, Journal of Hazardous Materials, Volumen 186, Números 2-3, 28 De Febrero de 2011, Páginas 1560-1567 (2011)

48. Haitao Zhang, Shuxin Ouyang, Zhaosheng Li, Lifei Liu, Tao Yu, Jinhua Ye, Zhigang Zou.: Preparation, characterization and photocatalytic activity of polycrystalline $\mathrm{Bi}_{2} \mathrm{O}_{3} / \mathrm{SrTiO}_{3}$ composite powders, Journal of Physics and Chemistry of Solids, Volumen 67, Número 12, De Diciembre de 2006, Páginas 2501-2505 (2006)

49. Heera T.R., Cindrella L.: PbS/CoS-Pani composite semiconductor films, Materials Science in Semiconductor Processing, Volumen 14, Número 2, De Junio de 2011 (2011)

50. Koji Nakamae, Masaki Chikahisa, Hiromu Fujioka.: Estimation of electron probe profile from SEM image through wavelet multiresolution analysis for inline SEM inspection, Image and Vision Computing, Volumen 25, Número 7, 1 De Julio de 2007, Páginas 1117-1123 (2007)

51. Richard Wirth.: Focused Ion Beam (FIB) combined with SEM and TEM: Advanced analytical tools for studies of chemical composition, microstructure and crystal structure in geomaterials on a nanometre scale, Chemical Geology, Volumen 261, Números 3-4, 30 De Abril de 2009, Páginas 217-229 (2009)

52. Qingyao Wang, Shengsong Ge, Qian Shao, Yuhua Zhao.: Self-assembly of $\mathrm{Sb}_{2} \mathrm{O}_{3}$ nanowires into microspheres: Synthesis and characterization, Physica B: Condensed Matter, Volumen 406, Número 4, 15 De Febrero de 2011, Páginas 731-736 (2011)

53. Juan Lu, Qiaofeng Han, Xujie Yang, Lude Lu, Xin Wang: Microwave-assisted synthesis and characterization of $3 \mathrm{D}$ flower-like $\mathrm{Bi}_{2} \mathrm{~S}_{3}$ superstructures, Materials Letters, Volumen 61, Números 14-15, De Junio de 2007 (2007)

54. Amir Abbas Rafati, Ahmad Reza Afraz Borujeni, Mojgan Najafi, Ahmad Bagheri.: Ultrasonic/surfactant assisted of CdS nano hollow sphere synthesis and characterization, Materials Characterization, Volumen 62, Número 1, De Enero de 2011, Páginas 94-98 (2011)

55. Mousavi S.H., Haratizadeh H., Kitai A.H.: Formation and characterization of zinc oxide nanowires grown on hexagonal-prism microstructures, Materials Letters, Volumen 65, Números 15-16, De Agosto de 2011, Páginas 2470-2472 (2011)

56. Shou-Nian Ding, Dan Shan, Huai-Guo Xue, Serge Cosnier.: A promising biosensingplatform based on bismuth oxide polycrystalline-modified electrode: Characterization and its application in development of amperometric glucose sensor, Bioelectrochemistry, Volumen 79, Número 2, De Octubre de 2010, Páginas 218-222 (2010)

57. Nasrin Talebian, Mohammad Reza Nilforoushan, Elahe Badri Zargar.: Enhanced antibacterial performance of hybrid semiconductor nanomaterials: $\mathrm{ZnO} / \mathrm{SnO} 2$ nanocomposite thin films, Applied Surface Science, Volumen 258, Número 1, 15 De Octubre de 2011, Páginas 547-555 (2011)

58. Fabio Bolzoni, Patrizia Fassina, Gabriele Fumagalli, Sara Goidanich.: Electrochemical characterization of corrosion resistant alloys in chloride solutions, In: Philippe Marcus and Vincent Maurice, Editor(s), Passivation of Metals and Semiconductors, and Properties of Thin Oxide Layers, Elsevier Science, Amsterdam, 2006, Páginas 573-578 (2006) 
59. Ankit Goyal, Kachhwaha S.: ZnO thin films preparation by spray pyrolysis and electrical characterization, Materials Letters, Volumen 68, 1 De Febrero de 2012, Páginas 354-356 (2012)

60. Libing Feng, Aihua Liu, Mei Liu, Yuying Ma, Jing Wei, Baoyuan Man.: Synthesis, characterization and optical properties of flower-like $\mathrm{ZnO}$ nanorods by non-catalytic thermal evaporation, Journal of Alloys and Compounds, Volumen 492, Números 1-2, 4 De Marzo de 2010, Páginas 427-432 (2010)

61. Yongjuan Lu, Gewen Yi, Junhong Jia, Yongmin Liang.: Preparation and characterization of patterned copper sulfide thin films on n-type $\mathrm{TiO}_{2}$ film surfaces, Applied Surface Science, Volumen 256, Número 23, 15 De Septiembre de 2010, Páginas 7316-7322 (2010) 